\title{
Determinando el pronóstico del paciente gran quemado: Correlación del Î́ndice de Garcés con el Abbreviated Burn Severity Index (ABSI)
}

\author{
Roberto Macchiavello M. ${ }^{1}$, María Angélica Paulos ${ }^{2}$, Carolina Soto D. ${ }^{1}$, \\ Manuel Calcagno L. ${ }^{1}$ y Carlos Barril ${ }^{3}$
}

Centro de Referencia Nacional del Gran Quemado, Hospital de Urgencia Asistencia Pública (HUAP)

Departamento de Cirugí Plástica Hospital Roberto del Río.

${ }^{3}$ Pontificia Universidad Católica de Chile. Santiago, Chile.

Recibido 2020-11-07, aceptado 2021-02-16

Correspondencia a: Dr. Roberto Macchiavello M. robertomac89@gmail.com

\section{Defining the prognosis of the burned patient: Correlation of the Garces Index with the Abbreviated Burn Severity Index (ABSI)}

Introduction: There are different prognostic scores to determine the probability of death in burned patients. The Garces Index (GI) used in Chile is not accepted worldwide. Aim: We decided to evaluate the correlation between IG and Abbreviated Burn Severity Index (ABSI), which is widely used. Materials and Method: Cross-sectional analytical and descriptive study of burned patients uploaded to the online platform "National Burns Registry" and then admitted to our center between July 2017 and July 2018. Pearson's correlation coefficient between IG and ABSI was calculated. Both variables were normalized to be able to make them comparable and the agreement between them was calculated with Lin's correlation coefficient. With logistic regression analysis, the associated probability of death was calculated for both variables and areas of the ROC curves of both scores were compared. Results: Of 141 patients, 15 died during hospitalization, with a mean GI $104 \pm 49$ and ABSI $7 \pm 2$. Pearson's correlation coefficient between GI and ABSI showed a very high correlation with a value of 0.928 . Lin's correlation coefficient gave moderate agreement with a value of 0.93 . When checking which of the two scores best predicts the probability of death through sensitivity and specificity analysis, the ROC curve covers more area in IG ( 0.82 vs 0.83$)$ without being statistically significant. Conclusions: Both scores, IG and ABSI, are very similar when it comes to predicting the probability of death, which is why they are comparable at the time of sharing the results in scientific studies.

Keywords: burns; mortality; prognosis; smoke inhalation injury.

\section{Resumen}

Introducción: Existen distintos scores pronósticos para determinar probabilidad de muerte en pacientes quemados. El Índice de Garcés (IG) utilizado en Chile, no es aceptado mundialmente. Objetivo: Decidimos evaluar la correlación entre IG y Abbreviated Burn Severity Index (ABSI), de uso generalizado. Materiales y Método: Estudio descriptivo analítico transversal de pacientes quemados subidos a la plataforma online "Registro Nacional de Quemados" y luego ingresados a nuestro centro entre julio de 2017 y julio de 2018. Se calculó coeficiente de correlación de Pearson entre IG y ABSI. Se normalizan ambas variables para hacerlas comparables y se calculó concordancia entre estas con coeficiente de correlación de Lin. Con análisis de regresión logística se calculó probabilidad de fallecer asociada para ambas variables y se compararon áreas de las curvas ROC de ambos scores. Resultados: De 141 pacientes, 15 fallecieron durante hospitalización, con medias de IG de $104 \pm 49$ puntos y ABSI $7 \pm 2$. El coeficiente de correlación de Pearson entre IG y ABSI demostró ser muy alto con un valor de 0,928. El coeficiente de correlación de Lin entregó concordancia moderada con un valor de 0,93 . Al comprobar cuál de las dos puntuaciones predice mejor la probabilidad de muerte mediante análisis de sensibilidad y especificidad, la curva ROC cubre más área en IG (0,82 vs. 0,83), sin ser estadísticamente significativo. Conclusiones: Ambos índices, IG y ABSI, son muy similares para predecir la probabilidad de muerte, por lo cual son equiparables a la hora de compartir resultados de estudios científicos.

Palabras clave: quemados; mortalidad; pronóstico; injuria inhalatoria. 


\section{Introducción}

El paciente gran quemado es aquel que requerirá tratamiento en unidades de quemados y/o unidades de paciente crítico (intermedio, intensivo). Están incluidos en esta definición todos los pacientes que cumplan con los criterios definidos en la Guías Clínicas GES ${ }^{1}$ : Índice de gravedad de Garcés mayor a 70 puntos, quemaduras $\mathrm{AB}$ o $\mathrm{B}$ mayor a $20 \%$ de la superficie corporal, mayor de 65 años con $10 \%$ o más de quemadura $\mathrm{AB}$ o $\mathrm{B}$, lesión de la vía aérea, quemaduras eléctricas por alta tensión, quemados politraumatizados, quemados con patologías graves asociadas o quemaduras profundas en zonas especiales (cara, cuello, manos, pies, pliegues articulares, genitales, mamas, axila).

La supervivencia de estos pacientes ha mejorado constantemente durante las últimas décadas. Con tasas generales de muerte en el rango de $5 \%$ a $15 \%$, la mortalidad todavía es la principal medida de resultado en la atención de quemaduras. Numerosos factores se combinan para entregarnos una imagen clínica pronóstica y así dictaminar las mejores intervenciones y resultados. Los distintos sistemas de puntuación (scores) tienen como objetivo utilizar el más predictivo de estos factores para generar una probabilidad de muerte esperada para un paciente determinado ${ }^{2}$. Además, pueden proporcionar puntos de referencia para la auditoría y la investigación.

Existen más de 45 modelos compuestos para predicción de mortalidad en lesiones térmicas, de las cuales solo unos pocos se han desarrollado con rigor metodológico ${ }^{3}$.

El Abbreviated Burn Severity Index (ABSI) o índice abreviado de gravedad de las quemaduras, descrito en 1982, es una escala mundialmente utilizada y aceptada de cinco variables, que ayuda a evaluar la gravedad de las quemaduras. Las variables consideradas son sexo, edad, presencia de injuria inhalatoria, presencia de una quemadura de espesor total y porcentaje de la superficie corporal total quemada (Tabla 1), cada una de estas variables otorga un puntaje que sumados indican la severidad y probabilidad de sobrevida estimada del paciente ${ }^{4}$.

El sistema de puntuación pronóstica más utilizado en Chile es el Î́ndice de Garcés (IG) desarrollado por el Dr. Mario Garcés en nuestro centro de referencia en la década de los $70^{5}$. Posteriormente en 1990, el Dr. René Artigas modificó el IG para ser utilizado con la población pediátrica. Este índice ha tenido una gran aceptación nacional desde entonces y se utiliza rutinariamente en todo nuestro país. También se usa comúnmente para compartir informes y resultados epidemiológicos nacionales en reuniones y artículos científicos. Por ello, en el 2007 las Garantías Explícitas de Salud en el manejo del gran quemado lo establecieron como uno de los criterios de derivación para el ingreso y traslado al Centro de Referencia Nacional. las:

El IG se calcula aplicando las siguientes fórmu-

Pacientes mayores de 20 años $=$ Edad +

(\% superficie corporal total quemaduras A x 1) + (\% superficie corporal total quemaduras $\mathrm{AB} \times 2)+$

(\% superficie corporal total quemaduras B x 3 )

Pacientes de 2 a 20 años (modificación Artigas) $=$ $(40$ - edad $)+(\%$ superficie corporal total

quemaduras A x 1) + (superficie corporal total quemaduras $\mathrm{AB} \times 2)+(\%$ superficie corporal total quemaduras $\mathrm{B} \times 3$ )

Tabla 1. Abbreviated Burn Severity Index (ABSI)

\begin{tabular}{|c|c|c|}
\hline Parámetro & Hallazgos & Puntaje \\
\hline Sexo & $\begin{array}{l}\text { Mujer } \\
\text { Hombre }\end{array}$ & $\begin{array}{l}1 \\
0\end{array}$ \\
\hline Edad (años) & $\begin{array}{c}0-20 \\
21-40 \\
41-60 \\
61-80 \\
81-100\end{array}$ & $\begin{array}{l}1 \\
2 \\
3 \\
4 \\
5\end{array}$ \\
\hline Injuria inhalatoria & $\begin{array}{l}\text { Sí } \\
\text { No }\end{array}$ & $\begin{array}{l}1 \\
0\end{array}$ \\
\hline $\begin{array}{l}\text { Presencia de quemadura de } \\
\text { espesor total }\end{array}$ & $\begin{array}{l}\text { Sí } \\
\text { No }\end{array}$ & $\begin{array}{l}1 \\
0\end{array}$ \\
\hline $\begin{array}{l}\% \text { Superficie corporal total } \\
\text { quemada }\end{array}$ & $\begin{array}{c}1-10 \\
11-20 \\
21-30 \\
31-40 \\
41-50 \\
51-60 \\
61-70 \\
71-80 \\
81-90 \\
91-100\end{array}$ & $\begin{array}{c}1 \\
2 \\
3 \\
4 \\
5 \\
6 \\
7 \\
8 \\
9 \\
10\end{array}$ \\
\hline $\begin{array}{l}\text { Puntaje ABSI y predicción } \\
\text { ABSI }\end{array}$ & Amenaza a la vida & $\begin{array}{c}\text { Probabilidad de } \\
\text { sobrevida (\%) }\end{array}$ \\
\hline $\begin{array}{l}2-3 \\
4-5\end{array}$ & $\begin{array}{l}\text { Muy baja } \\
\text { Moderada }\end{array}$ & $\begin{array}{c}\geq 99 \\
98\end{array}$ \\
\hline $6-7$ & Moderadamente severa & $80-90$ \\
\hline $8-9$ & Seria & $50-70$ \\
\hline $10-11$ & Severa & $20-40$ \\
\hline$>12$ & Máxima & $\leq 10$ \\
\hline
\end{tabular}


Pacientes menores de 2 años (modificación Artigas $)=(40-$ edad $)+(\%$ superficie corporal total quemaduras A x 2$)+(\%$ superficie corporal total quemaduras $\mathrm{AB} \times 2)+(\%$ superficie corporal total quemaduras B x 3) +20

Los pacientes quemados se clasifican según su puntuación en: 21-40 puntos

Leve: sin riesgo vital.

41-70 puntos Moderado: sin riesgo vital, salvo complicaciones.

71-100 puntos Grave: probabilidad de muerte menor que supervivencia. Mortalidad $<30 \%$.

101-150 puntos Crítico: Mortalidad 30\%-50\%.

$>150$ puntos Supervivencia excepcional: Mortalidad $>50 \%$.

Como sabemos, el IG solo se usa en Chile y en algunos otros países de América Latina, pero no es conocido ni aceptado mundialmente. Decidimos evaluar la correlación entre el IG y el ABSI, que tiene un uso generalizado. El objetivo de este estudio es determinar el grado de correlación entre estos dos puntajes, y así tener fundamentos para utilizar nuestro índice nacional en informes internacionales.

\section{Materiales y Método}

Desarrollamos un estudio descriptivo y analítico transversal de todos los pacientes grandes quemados que fueron subidos a la plataforma online de gestión de derivación y traslados, el "Registro Nacional de Quemados"y luego ingresados en nuestro centro de referencia entre julio de 2017 y julio de 2018, sin casos perdidos $(n=141)$. Este universo no incluyó a los pacientes menores de 15 años, ya que estos pacientes están excluidos del Registro Nacional de Quemados por ser tratados en las unidades de quemados de los hospitales pediátricos. Todos los datos fueron debidamente solicitados y autorizados por las autoridades correspondientes del Ministerio de Salud de Chile. Se calculó el coeficiente de correlación de Pearson entre el Índice de Gravedad de Garcés y el ABSI. Se normalizaron ambas variables para poder hacerlas comparables y se calculó la concordancia entre estas variables con el coeficiente de correlación de Lin, con la siguiente formula ([x$\mathrm{X}] / \mathrm{s}$ ). Con análisis de regresión logística se calculó la probabilidad de fallecer asociada para ambas variables y se compararon las áreas de las curvas ROC (Receiver Operating Characteristic) para los Índices de Garcés e ABSI. Los análisis estadísticos se realizaron con el programa Stata ${ }^{\circledR}$ versión 11.2 (Stata Corporation, College Station, Texas).

\section{Resultados}

De un total de 141 pacientes, 15 fallecieron durante la hospitalización, con una media de IG de $104 \pm 49$ puntos y un ABSI promedio de $7 \pm 2$ (Tabla 2).

El coeficiente de correlación de Pearson entre IG y ABSI demostró una muy alta correlación entre ambas variables con un valor de 0,928 e intervalo de confianza del 95\% entre 0,904 y 0,947 (Figura 1). Al normalizar ambas variables el coeficiente de correlación de Lin entrega una concordancia moderada con un valor de 0,93 con un intervalo de confianza del $95 \%$ entre 0,904 y 0,951 (Figura 2). La concordancia disminuye en valores de $\mathrm{IG}>180$ y $\mathrm{ABSI}>12$.

Respecto a la proporción de variabilidad que es no explicada por estos modelos pronósticos, ambos se ajustan bien teniendo pseudo R2 muy parecidos (IG 0,20 y ABSI 0,22), pero no explican por si solos toda la probabilidad de morir de un paciente gran quemado. Pudimos comprobar cuál de las dos puntuaciones predice mejor la probabilidad de muerte, mediante el análisis de sensibilidad y especificidad. La curva ROC cubre más área en el caso del Índice de Garcés $(0,82$ vs. 0,83$)$, pero esta diferencia no es estadísticamente significativa (Figura 3).

\section{Discusión}

La mortalidad del paciente Gran Quemado es todavía la principal medida de resultado en la atención de los mismos. Existen múltiples scores de severidad descritos, siendo el Abbreviated Burn Severity Index (ABSI) uno de los más aceptados y utilizados. En Chile, en cambio, el Índice de Garcés (IG) sigue siendo el más difundido tanto en la clínica, como en la generación de políticas públicas (GES) e investigación. Un ejemplo de esto último es la utilización del Índice de Garcés para el análisis comparativo de mortalidad frente a diferentes estrategias terapéuticas, como lo hace Villegas ${ }^{6}$, al presentar los resultados del proceso de modernización y protocolización del manejo de pacientes quemados en nuestra unidad. En este contexto, se hacía necesario intentar validar nuestro score con alguno utilizado globalmente.

Es importante la constante revisión de los scores empleados en la clínica del paciente quemado, 


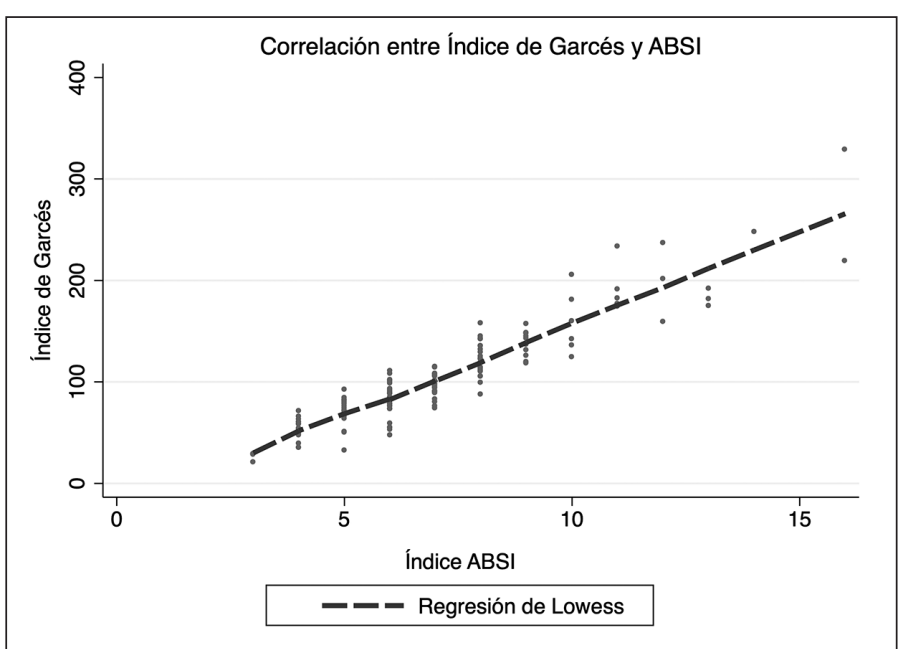

Figura 1. Correlación entre el Índice de Garcés (IG) y ABSI. La regresión logística de Lowess demostró muy buena correlación entre ambas variables (IG y ABSI)

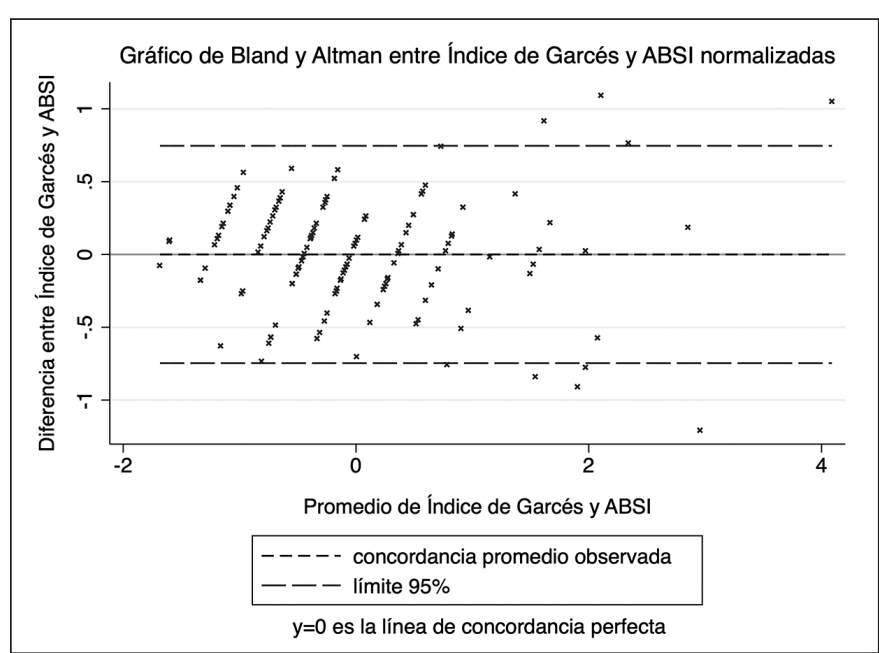

Figura 2. Gráfico de Bland-Altman para el Índice de Garcés (IG) y ABSI. La concordancia entre las variables normalizadas es excelente, muy cercana a +1 . En quemaduras con IG y ABSI más altos, hay menor concordancia.

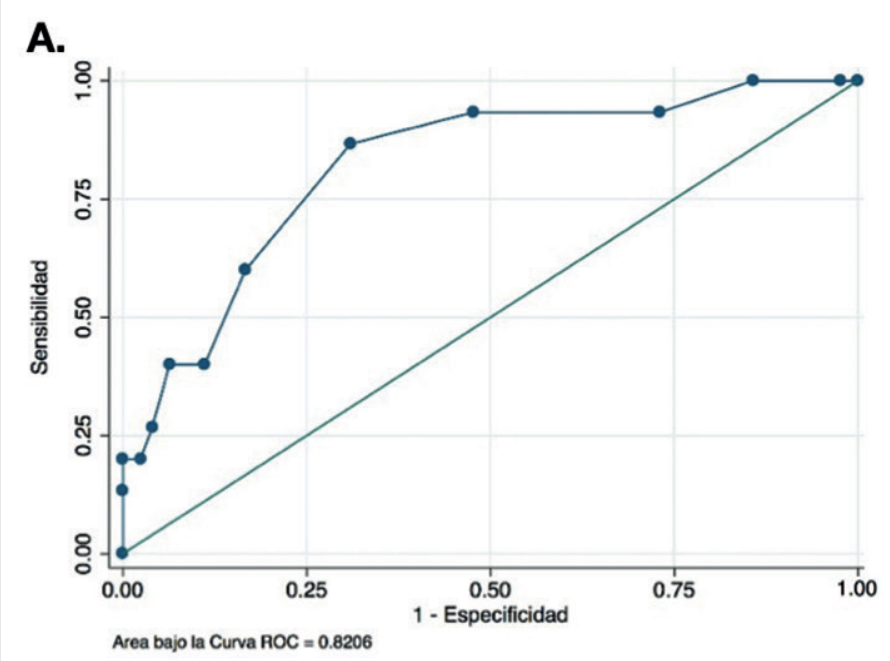

B.

Figura 3. Curvas ROC para el Índice de Garcés (IG) y ABSI. A: Curva ROC ABSI. B: Curva ROC IG. La curva ROC cubre más área en el caso del IG, pero no es estadísticamente significativa.

ya que de eso dependen una serie de decisiones clínicas y de gestión importantes. En un reciente estudio alemán publicado en la revista Burns ${ }^{7}$, se hicieron la pregunta de si el ABSI todavía predecía con precisión supervivencia del paciente quemado. Los datos de 14.984 pacientes del German Burns Registry (símil a nuestro Registro Nacional de Quemados) del 2015 al 2018 fueron analizados para reevaluar las variables incluidas en el ABSI, identificar discrepancias entre la probabilidad de supervivencia predicha relacionada con la edad y la tasa de supervivencia real. Concluyeron que el ABSI original no predecía con precisión la supervivencia en la cohorte actual. En particular, el análisis univariado identificó la edad, la superficie corporal total quemada, el espesor total de las quemaduras y la injuria inhalatoria como factores importantes que afectan la supervivencia. El sexo no resultó ser una variable significativa $y$, por lo tanto, fue excluido de la puntuación modificada que propusieron. El sexo, como hemos visto, no está considerado como variable en el Índice de Garcés. 


\section{ARTÍ́CULO ORIGINAL}

En nuestro caso pudimos demostrar que ambos índices, IG y ABSI, son muy similares a la hora de predecir la probabilidad de muerte, por lo cual son equiparables a la hora de compartir resultados de estudios científicos. De todas maneras, sugerimos que el Índice de Garcés se puede optimizar al incluir nuevas variables relevantes como la injuria inhalatoria (presente en ABSI) que afecta hasta el 33\% de los pacientes grandes quemados ${ }^{8} \mathrm{o}$ el índice de masa corporal (IMC), el cual podría estar relacionado con la mortalidad en pacientes quemados ${ }^{9}$, lo cual puede ser motivo de estudios futuros.

\section{Responsabilidades éticas}

Protección de personas y animales. Los autores declaran que para esta investigación no se han realizado experimentos en seres humanos ni en animales.

Confidencialidad de los datos. Los autores declaran que en este artículo no aparecen datos de pacientes.

Conflictos de interés: no hay.

\section{Bibliografía}

1. Ministerio de Salud de Chile. Guías Clínicas AUGE Gran Quemado. 2016

[Consultado el 7 de febrero 2020]

Disponible en: http://www.bibliotecaminsal.

cl/wp/wp-content/uploads/2016/04/ GPC-GRAN-QUEMADO-FINAL-18MARZO-2016 DIAGRAMADA.pdf

2. Sheppard NN, Hemington-Gorse S, Shelley OP, Philp B, Dziewulski P. Prognostic scoring systems in burns: a review. Burns. 2011;37:1288-95.

3. Woods JF, Quinlan CS, Shelley OP. Predicting Mortality in Severe BurnsWhat Is the Score? Plast Reconstr Surg
Glob Open 2016;4:e606. doi: 10.1097/ GOX.0000000000000584.

4. Tobiasen J, Hiebert JM, Edlich RF. The abbreviated burn severity index. Ann Emerg Med. 1982;11:260-2.

5. Garcés M. Bases clínicas y epidemiológicas para un pronóstico del enfermo quemado. Cuad Med Soc. 1975;1:9-14.

6. Villegas J, Torres E, Pedreros C, Singh P, Longton C, Said JC, et al. Mortalidad tras un año de protocolización en el manejo del paciente quemado. Rev Chil Cir. 2010;62:144-9.

7. Bartels P, Thamm O, Elrod J, Fuchs $\mathrm{P}$, Reinshagen $\mathrm{K}$. The ABSI is dead, long live the ABSI - reliable prediction of survival in burns with a modified Abbreviated Burn Severity Index. Burns 2020;46:1272-9.

8. Chung KK, Rhie RY, Lundy JB, Cartotto R, Henderson E, Pressman MA, et al. A Survey of Mechanical Ventilator Practices Across Burn Centers in North America. J Burn Care Res. 2016;37:e1319.

9. Tapking C, Houschyar KS, Rontoyanni VG, Hundeshagen G, Kowalewski KF, Hirche C, et al. The Influence of Obesity on Treatment and Outcome of Severely Burned Patients. J Burn Care Res. 2019;40:996-1008. 